\title{
AN EXTENSION OF THE ERDŌS-SZEKERES THEOREM ON LARGE ANGLES
}

\author{
I. BÁRÁNY \\ Received 28 October 1985
}

The existence of a function $n(\varepsilon) \quad(\varepsilon>0)$ is established such that given a finite set $V$ in the plane there exists a subset $W \subseteq V,|W|<n(\varepsilon)$ with the property that for any $v \in V \backslash W$ there are two points $w_{1}, w_{2} \in W$ such that the angle $\varangle\left(w_{1} v w_{2}\right)>\pi-\varepsilon$.

\section{Results}

The Erdós-Szekeres theorem [5] mentioned in the title says that if $V \subset \mathbf{R}^{2}$ and $|V| \geqq 2^{n}$, then $V$ contains three points $a, b, c \in V$ such that $\varangle(a b c)>\pi(1-(1 / n))$. Here $\Varangle(a b c)$ denotes the angle at vertex $b$ of the triangle $a b c$, thus $0 \leqq \varangle(a b c) \leqq \pi$.

The aim of this paper is to extend this theorem in the following way.

Theorem 1. For any $\varepsilon>0$ there exists $n(\varepsilon)$ such that every finite set $V \subset \mathbf{R}^{2}$ contains a subset $W \subseteq V,|W| \leqq n(\varepsilon)$ with the property that for any $v \in V \backslash W$ there are points $w_{1}, w_{2} \in W$ such that $\Varangle\left(w_{1} v w_{2}\right)>\pi-\varepsilon$.

In other words, every finite set $V \subset \mathbf{R}^{2}$ contains a "small" subset $W$ from which any point of $V \backslash W$ is seen at a "large" angle.

We will show that one can take

$$
n(\varepsilon)=\left(\frac{c_{1}}{\varepsilon}\right)^{\left(c_{\mathrm{g}} / \varepsilon\right)}
$$

where $c_{1}$ and $c_{2}$ are contants. On the other hand $n(\varepsilon) \geqq 2^{c / \varepsilon}$ since one can construct a set $V \subset \mathbf{R}^{2}$ with $|V|=2^{c / s}$ such that $\Varangle(a b c) \leqq \pi-\varepsilon$ for every $a, b, c \in V$ (see [6]). The proof-method of Theorem 1 works in $\mathbf{R}^{d}(d>2)$ as well giving

Theorem 2. For any $\varepsilon>0$ and $d \geqq 2$ there exists a constant $n(d, \varepsilon)$ such that every finite set $V \subset \mathbf{R}^{d}$ contains a subset $W \subseteq V,|W| \leqq n(d, \varepsilon)$ with the property that for any $v \in \bigvee W$ there are points $w_{1}, w_{2} \in W$ with $\varangle\left(w_{1} v w_{2}\right)>\pi-\varepsilon$.

Let $n_{0}(d, \varepsilon)$ be the smallest number $n(d, \varepsilon)$ for which Theorem 2 holds. When 
$\varepsilon<1$, say, we obtain from the proof that

$$
2^{(c / \varepsilon)^{d-1}} \leqq n_{0}(d, \varepsilon) \leqq\left(\frac{c_{1} \sqrt{d}}{\varepsilon}\right)^{\left(c_{2} / \varepsilon\right)^{d-1}}
$$

where for the lower bound see [1] or [6].

In the proof of Theorem 1 and 2 we may assume that $\varepsilon>0$ is small because if the theorem holds with some $\varepsilon>0$ then it holds with every $\varepsilon^{\prime} \geqq \varepsilon$ : one can take simply $n\left(d, \varepsilon^{\prime}\right)=n(d, \varepsilon)$. So the above estimation for $n_{0}(d, \varepsilon)$ holds for small $\varepsilon$. When $\varepsilon=\pi / 2$, one can do better. More precisely, let $\varepsilon \geqq \operatorname{arc} \cos (1 / d)$. Then a stronger version of Theorem 2 is true, which was also proved by János Pach independently [7].

Theorem 3. For every $d=2,3, \ldots$ there exists a number $n(d)$ such that the following holds. Every finite set $V \subset \mathbf{R}^{d}$ contains a subset $W \leqq V,|W| \leqq n(d)$ such that $W \subseteq$ boundary (conv $V)$ and for any point $v \in(\operatorname{conv} V) \backslash \bar{W}$ there are points $w_{1}, w_{2} \in$ $\in \bar{W}$ with $\cos \measuredangle\left(w_{1} v w_{2}\right) \leqq-1 / d$.

From the proof of this theorem we get that for $\arccos (1 / d)<\varepsilon<2 \pi, n_{0}(d, \varepsilon) \leqq$ $\leqq 2^{d(d-1)} \cdot d^{d(d+1)}$. Erdős and Füredi [4] gave an example $V \subset \mathbf{R}^{d}$ with $|V| \geqq c_{\alpha}^{d}$ points such that $\Varangle(a b c)<\alpha$ for every $a, b, c \in V$, where $\alpha>\pi / 3$ is fixed and $c_{\alpha}>1$. This shows that for $\operatorname{arc} \cos (1 / d)<\varepsilon<2 \pi, c_{\pi-\varepsilon}^{d} \leqq n_{0}(d, \varepsilon)$.

The reason for the bound arc cos $(1 / d) \leqq \varepsilon$ in Theorem 3 is the following fact which is certainly well-known. If the point $a$ belongs to a $d$-dimensional simplex, then the simplex has two vertices $b$ and $c$ with $\Varangle(b a c) \geqq \pi-\operatorname{arc} \cos (1 / d)$.

I mention that our theorems hold for compact sets $V \subset \mathbf{R}^{d}$ (instead of finite). This can be seen using a simple continuity argument.

In [5] Erdôs and Szekeres dealt with a related problem. Having fixed a basis in $\mathbf{R}^{d}$, a box is defined as $\left\{x \in \mathbf{R}^{d}: a_{i} \leqq x_{i} \leqq b_{i} i=1, \ldots, d\right\}$. Now Erdős and Szekeres show in [5] that if $V \subset \mathbf{R}^{d},|V|>2^{2^{d-1}}$, then there are points $a, b, c \in \mathbf{R}^{d}$ such that $b$ is contained in the smallest box containing $a$ and $c$. This result is extended in a paper by Bárány and Lehel [2] in the same way as Theorem 1 here extends the ErdósSzekeres theorem on large angles. In particular, Theorem 2 follows from the results of [2] but with a much weaker estimation on $n_{0}(d, \varepsilon)$.

\section{Sketch of the proof of Theorem 1}

The proof of Theorem 1 will be algorithmic. In this section we give an informal description of the algorithm together with some preliminary definitions and lemmas. We will work with small squares whose sides are parallel to the coordinate axes. $\varrho(x, y)$ denotes the Euclidean distance of $x, y \in \mathbf{R}^{2}$.

Definition 1. We say that $s_{1}, s_{2} \in \mathbf{R}^{2}$ cover the square $C \subseteq \mathbf{R}^{2}$ if $\Varangle\left(s_{1} c s_{2}\right)>\pi-\varepsilon$ for every $c \in C$.

Let $C^{0}$ be the smallest square containing $V$. The algorithm will find a subdivision of $C^{0}$ into small squares and a set $W \subset V,|W| \leqq n(\varepsilon)$ whose pairs cover every small square $C$ in the subdivision provided $C \cap(V \backslash W) \neq \emptyset$. 
Definition 2. $\left(C_{A}, S\right)$ is called a good pair if $C_{A}$ is a square of side length $\Delta$ and $S \subset \mathbf{R}^{2}$ with the properties

(i) $\varrho(c, s) \geqq 10 \sqrt{2} \Delta \varepsilon^{-1} \quad(\forall c \in C, \forall s \in S)$,

(ii) $\varangle\left(s_{1} c s_{2}\right) \geqq 0,5 \varepsilon \quad\left(\forall c \in C, \forall s_{1}, s_{2} \in S, s_{1} \neq s_{2}\right)$.

Sometimes we shall omit the index $\Delta$ and simply say that $(C, S)$ is a good pair. A good pair can be thought of as a square $C$ together with a set of directions $S$ because, by condition (i), the points in $S$ are far away from $C$ (almost at infinity with respect to its diameter). As a rule, $S$ will be a subset of $V$ and the algorithm will produce good pairs $(C, S)$ in such a way that some point $s \in S$ together with a suitable point $v \in V$ will cover $C$. Property (ii) means that the directions in $S$ are not too close to each other. This fact implies at once a simple property of good pairs:

Lemma 1. If $(C, S)$ is a good pair, then $|S| \leqq 4 \pi \varepsilon^{-1}$.

Definition 3. The good pair $\left(C_{A}^{1}, S\right)$ covers the square $C_{\Delta}^{2}$ if there is an $s \in S$ such that for every $c \in C_{\Delta}^{1}, c$ and $s$ cover $C_{\Delta}^{2}$.

The crucial property of good pairs is given next.

Lemma 2. If $\left(C_{\Delta}^{1}, S\right)$ and $\left(C_{\Delta}^{2}, S\right)$ are good pairs and

$$
\begin{gathered}
0.1 \varrho\left(c_{2}, s\right) \geqq \varrho\left(c_{1}, c_{2}\right) \quad\left(\forall c_{1} \in C_{\Delta}^{1}, \forall c_{2} \in C_{\Delta}^{2}, \forall s \in S\right), \\
\varrho\left(c_{1}, c_{2}\right) \geqq 10 \sqrt{2} \Delta \varepsilon^{-1} \quad\left(\forall c_{1} \in C_{\Delta}^{1}, \forall c_{2} \in C_{\Delta}^{2}\right),
\end{gathered}
$$

then either $\left(C_{\Delta}^{1}, S\right)$ covers $C_{\Delta}^{2}$, or $\left(C_{\Delta}^{1}, S \cup\{u\}\right)$ is a good pair for every $u \in C_{\Delta}^{2}$.

We can now explain how the algorithm works. At a certain stage we will have a good pair $\left(C_{A}, S\right)$ such that no $s_{1}, s_{2} \in S$ cover $C_{A}$. We then subdivide $C_{A}$ by a set of smaller squares of side length $\Delta^{\prime}=2^{-k} \Delta(k=k(\varepsilon)$ will be specified later). A small square $C_{\Delta^{\prime}}$ of this subdivision is called a cell if $C_{d^{\prime}} \cap V \neq \emptyset$. We pick one point $u \in V$ from each cell $C_{A^{\prime}}$. These points form a set $U \subseteq V$ with $|U| \leqq 4^{k}$. If $C_{A^{\prime}}$ is a cell then $\left(C_{A^{\prime}}, S\right)$ is clearly a good pair. Now the main part of the algorithm can be named as "either cover $C_{4}$ " or augment $S$ ". More precisely, on applying Lemma 2 to the good pairs $\left(C_{\Delta^{\prime}}^{1}, S\right)$ and $\left(C_{A^{\prime}}^{2}, S\right)$ one finds a point $u \in U$ such that either $C_{A^{\prime}}^{1}$, is covered by $u$ and a suitable $s \in S$ or $\left(C_{\Delta^{\prime}}^{1}, S \cup\{u\}\right)$ is a good pair, unless a very special case occurs. If this special case does not come up then, in view of Lemma 1, we are finished after at most $4 \pi \varepsilon^{-1}$ steps of the type "either cover or augment". In this case the set $W$ will be the union of all the sets $U$ and will have cardinality at most $\left(4^{k}\right)^{4 \pi \varepsilon^{-1}}$. The special case which needs special care as well corresponds to, roughly speaking, a point $v \in V$ such that $\varangle\left(v_{1} v v_{2}\right) \leqq \pi-\varepsilon$ for every $v_{1}, v_{2} \in V$. Such a point $v$ must be contained in $W$, so we cannot choose $u \in C_{\Delta^{\prime}} \cap V$ arbitrarily if $v \in C_{\Delta}^{\prime}$ as we could in the other case.

To close this section we prove Lemmas 1 and 2 .

Proof of Lemma 1. Consider a point $c \in C$ and a unit circle $E$ around it. For each $s \in S$ define the $\operatorname{arc} A_{s}$ as $A_{s}=\{x \in E: \Varangle(x c s)<0.25 \varepsilon\}$. These arcs are pairwise disjoint by (ii) so the sum of their lengths is at most $2 \pi$ :

$$
2 \pi \geqq \sum_{s \in S} \text { length }\left(A_{s}\right)=|S| \cdot 0.5 \varepsilon \text {. }
$$


Proof of Lemma 2. Assume first that there is an $s \in S$ such that $\varangle\left(c_{2} c_{1} s\right) \leqq 0.9 \varepsilon$ $\left(\forall c_{1} \in C^{1}, \forall c_{2} \in C^{2}\right)$. Then the condition $0.1 \varrho\left(c_{2}, s\right) \geqq \varrho\left(c_{1}, c_{2}\right)$ implies

and then

$$
\frac{\sin \varangle\left(c_{2} c_{1} s\right)}{\sin \varangle\left(c_{2} s c_{1}\right)}=\frac{\varrho\left(c_{2}, s\right)}{\varrho\left(c_{2}, c_{1}\right)} \geqq 10,
$$

$$
\sin \varangle\left(c_{2} s c_{1}\right) \leqq 0.1 \sin \varangle\left(c_{2} c_{1} s\right) \leqq 0.1 \sin 0.9 \varepsilon<\sin 0.1 \varepsilon .
$$

This shows that in this case $\varangle\left(c_{1} c_{2} s\right)>\pi-(0.9 \varepsilon+0.1 \varepsilon)=\pi-\varepsilon$, i.e., $\left(C^{1}, S\right)$ covers $C^{2}$ by definition.

Assume now that for each $s \in S$ there are $c_{1}^{0} \in C^{1}$ and $c_{2}^{0} \in C^{2}$ such that $\varangle\left(c_{2}^{0} c_{1}^{0} s\right)>0.9 \varepsilon$. Then, using the condition $\varrho\left(c_{1}, c_{2}\right) \geqq 10 \sqrt{2} \Delta \varepsilon^{-1}$, an easy argument shows that $\Varangle\left(c_{2} c_{1} s\right) \geqq 0.5 \varepsilon$ for each $c_{1} \in C^{1}, c_{2} \in C^{2}, s \in S$, i.e., $\left(C^{1}, S \cup\{u\}\right)$ is a good pair for every $u \in C^{2}$.

\section{The proof of Theorem 1}

We start with the good pair $\left(C^{0}, \varnothing\right)$ where $C^{0}$ is the smallest square containing $V$.

Assume that at a certain stage of the algorithm we have a good pair $\left(C_{\Delta}^{0}, S\right)$ with the property that no $s_{1}, s_{2} \in S$ cover $C_{\Delta}^{0}$. (If this were not so $C_{\Delta}^{0}$ would be covered.) Now we subdivide $C_{A}^{0}$ by smaller squares of side length $\Delta^{\prime}=2^{-k} A$, where $k=k(\varepsilon)$ will be specified later. Let $\mathscr{C}$ denote the set of cells of this subdivision, i.e., $C_{A^{\prime}} \in \mathscr{C}$ if $C_{A^{\prime}} \cap V \neq \emptyset$.

Now we try to apply the "either cover or augment" procedure. In order to do so, there are some cases to consider.

Case 1. For each $C \in \mathscr{C}$ there is a $C^{\prime} \in \mathscr{C}$ such that $\left(C^{\prime}, S\right)$ covers $C$.

In this case we pick one point $u \in V$ from each cell $C \in \mathscr{C}$. The set $U$ of these points satisfies $|U| \leqq|\mathscr{C}| \leqq 4^{k}$. Moreover, for every $C \in \mathscr{C}$ there are $u \in U$ and $s \in S$ such that $C$ is covered by $u$ and $s$.

Now we assume that there is a cell $C \in \mathscr{C}$ which is not covered by any pair $\left(C^{\prime}, S\right)$ with $C^{\prime} \in \mathscr{C}$. Such a cell is called uncovered.

Case 2. There are two uncovered cells $C_{1}, C_{2} \in \mathscr{C}$ with $\varrho\left(c_{1}, c_{2}\right) \geqq 1 / 6 \Delta$ for each $c_{1} \in C_{1}$ and $c_{2} \in C_{2}$.

Then we pick one point from each cell as in Case 1. The cells covered by some pair $(C, S)$ with $C \in \mathscr{C}$ will then be covered by a suitable $u \in U$ and $s \in S$.

Consider now a cell $C \in \mathscr{C}$ which is uncovered. Then either

$$
\varrho\left(c, c_{1}\right) \geqq \frac{1}{12} \Delta-\frac{1}{\sqrt{2}} \Delta^{\prime} \quad\left(\forall c \in C, \forall c_{1} \in C_{1}\right),
$$

or

$$
\varrho\left(c, c_{2}\right) \geqq \frac{1}{12} \Delta-\frac{1}{\sqrt{2}} \Delta^{\prime} \quad\left(\forall c \in C, \forall c_{2} \in C_{2}\right) .
$$


By symmetry we may and do assume that the first inequality holds. Let $u$ be the point picked from $C_{1}$.

Claim. (C, $S \cup\{u\})$ is a good pair provided $2^{k}>300 \varepsilon^{-1}$.

Proof. We are going to apply Lemma 2 to the good pairs $(C, S)$ and $\left(C_{2}, S\right)$. The first condition holds because for every $c \in C, c_{1} \in C_{1}$ and $s \in S$

$$
0.1 Q\left(c_{1}, s\right) \geqq 0.1 \min _{c^{0} \in C_{A}^{0}}\left(c^{0}, s\right) \geqq 0.1 \cdot 10 \sqrt{2} \Delta \varepsilon^{-1}>\sqrt{2} \Delta \geqq \varrho\left(c, c_{1}\right)
$$

(if $\varepsilon<1$, say). As for the second condition,

$$
\varrho\left(c, c_{1}\right) \geqq \frac{1}{12} \Delta-\frac{1}{\sqrt{2}} \Delta^{\prime}=\left(\frac{1}{12} 2^{k}-\frac{1}{\sqrt{2}}\right) \Delta^{\prime}
$$

and this has to be larger than $10 \sqrt{2} \Delta^{\prime} \varepsilon^{-1}$. This holds, for instance, when we choose $k=\left[\log _{2}\left(300 \varepsilon^{-1}\right)\right]$.

So the conditions of Lemma 2 hold. But $C_{1} \in \mathscr{C}$ is an uncovered cell and then the second alternative occurs: $(C, S \cup\{u\})$ is a good pair for every $u \in C_{1} \cap V$.

We can see now that in Case 2 the "either cover or augment" method works.

From now on we assume that there are uncovered cells in $\mathscr{C}$ and for any two uncovered cells $C_{1}, C_{2} \in \mathscr{C} \varrho\left(c_{1}, c_{2}\right) \leqq \Delta / 6+2 \sqrt{2} \Delta^{\prime} \quad\left(\forall c_{i} \in C_{i} i=1,2\right)$.

Let $K$ be (one of) the smallest square(s) $K \subset C_{A}^{0}$ which contains all uncovered cells from $\mathscr{C}$. The side length of $K$ is at most $\Delta / 6+2 \sqrt{2} \Delta^{\prime}$. Further, let $L \subset C_{A}^{0}$ be a square with side length $\Delta / 2$ containing $K$ and such that the minimal distance between $K$ and $C_{A}^{0} \backslash L$ is at least $10 \sqrt{2} A^{\prime} \varepsilon^{-1}$. We assume further that the sides of $L$ are contained in the lines defining the subdivision of $C_{4}^{0}$ into the smaller squares $C_{A^{\prime}}$. Such an $L$ exists if

$$
\left(\frac{1}{6} \Delta+2 \sqrt{2} \Delta^{\prime}\right)+10 \sqrt{2} \Delta^{\prime} \varepsilon^{-1}<\frac{1}{2} \Delta,
$$

which is again true if $k=\left[\log _{2}\left(300 \varepsilon^{-1}\right)\right]$.

Finally we define a partition of $\mathscr{C}$ :

$\mathscr{C}^{z}=\left\{C \in \mathscr{C}: C\right.$ is covered by some good pair $\left.\left(C^{\prime}, S\right), C^{\prime} \in \mathscr{C}\right\}$,

$\mathscr{C}^{x}=\left\{C \in \mathscr{C}: C\right.$ is uncovered and $(C, S)$ covers every cell in $\left.C_{\Delta}^{0} \backslash L\right\}$, $\mathscr{C}^{y}=\left(\mathscr{C} \backslash \mathscr{C}^{z} \cup \mathscr{C}^{x}\right)$.

Case $3 . \mathscr{C}^{x}=\emptyset$.

Again we pick one point $u \in V$ from each cell, these points form the set $U \subset V$. The cells in $\mathscr{C}^{z}$ will be covered by some $u \in U$ and $s \in S$ in the same way as in Case 1 . Let $C \in \mathscr{C}^{y}$ and consider a cell $C^{\prime} \in \mathscr{C}, C^{\prime} \subseteq C_{a}^{0} \backslash L$ which is not covered by the good pair $(C, S)$. Let $u \in C^{\prime} \cap U$.

Claim. $(C, S \cup\{u\})$ is a good pair.

The proof is almost identical with that of Case 2 and is omitted. works.

We see again that in this case the method of "either cover or augment" 
Case 4. $\quad \mathscr{C}^{x} \neq \emptyset$

In this case we do not pick any point from $V$ but make the promise to pick one point from the set $X_{1}=V \cap\left(\cup \mathscr{C}^{x}\right)$ at a later stage of the algorithm. For further reference we rename $K$ as $K_{1}, L$ as $L_{1}, \mathscr{C}, \mathscr{C}^{x}, \mathscr{C}^{y}, \mathscr{C}^{z}$ as $\mathscr{C}_{1}, \mathscr{C}_{1}^{x}, \mathscr{C}_{1}^{y}$ and $\mathscr{C}_{1}^{z}$. We observe that if we pick a point $u \in X_{1}$, then each $C \in \mathscr{C}_{1}, C \subseteq C_{\Delta}^{0} \backslash L_{1}$ will be covered by $u$ and a suitable $s \in S$. The algorithm continues by subdividing $L_{1}$ into $2^{k} \times 2^{k}$ smaller squares and considering $L_{1}$ as $C_{4}^{0}$ in the preceding step.

If Case 4 occurs, we get two squares $K_{2}$ and $L_{2}$, sets of cells $\mathscr{C}_{2}, \mathscr{C}_{2}^{x}, \mathscr{C}_{2}^{y}, \mathscr{C}_{2}^{x}$ (the sides of $L_{2}$ are of length $\Delta / 4$ ) and a new set of "promise" $X_{2}=V \cap\left(\cup \mathscr{C}_{2}^{x}\right)$. In this case we make the promise to pick a point from the set $X_{1} \cap X_{2}$ (if $X_{1} \cap X_{2} \neq \emptyset$ ) and continue in the same way as before. So we go on like that producing the squares $K_{i}, L_{i}$, sets of cells $\mathscr{C}_{i}, \mathscr{C}_{i}^{x}, \mathscr{C}_{i}^{y}, \mathscr{C}_{i}^{z}$ and sets of promises $X_{1} \cap X_{2} \cap \ldots \cap X_{i}$.

We can keep our promises only if $X_{1} \cap \ldots \cap X_{i} \neq \emptyset$. Suppose this is so. Again, we try to use the method of "either cover or augment" on the square $L_{i}$ (side-length $2^{-i} \Delta$ ). If it works (i.e. Cases 1,2 or 3 occur), we can keep our promise by choosing a point from $C \cap\left(X_{1} \cap \ldots \cap X_{i}\right)$ for a suitable $C \in \mathscr{C}_{i}$. If it does not work, then Case 4 occurs again: we get $X_{i+1} \neq \emptyset, K_{i+1}, L_{i+1}$, etc.

Case $4 a$. $\bigcap_{j=1}^{i} X_{t} \neq \emptyset$ but $\bigcap_{j=1}^{i+1} X_{i}=\emptyset$ for some $i=1,2,3, \ldots$.

Case $4 b . \bigcap_{j=1}^{i} X_{i} \neq 1 \emptyset$ for $i=1,2,3, \ldots$.

In Case $4 \mathrm{~b} \bigcap_{i=1}^{\infty} X_{i} \subseteq \bigcap_{i=1} L_{i}=\{u\} \subseteq V$ because the squares $L_{i}$ shrink to a single point. Then we pick the point $u$ and put it in the set $W$. It is easily checked that for every $i$ and every $C \in \mathscr{C}_{i}, C \subseteq C_{\lambda}^{0} \backslash L_{i}$ is covered by $u$ and a suitable point $s \in S$. ing rules:

In Case $4 \mathrm{a}$ we pick a point $u$ from each cell $C \in \mathscr{C}_{i+1}$ according to the follow-

$$
\begin{gathered}
u \in V \cap C, \text { if } C \cap \bigcap_{j=1}^{i} X_{j}=\emptyset \text { and } C \cap X_{i+1}=\emptyset, \\
u \in X_{i+1} \cap C, \text { if } C \cap X_{i+1} \neq \emptyset \\
u \in C \cap \bigcap_{j=1}^{i} X_{j} \text { if } C \cap \bigcap_{j=1}^{i} X_{j} \neq \emptyset .
\end{gathered}
$$

The points chosen this way form the set $U$, clearly $U \subseteq V$ and $|U| \leqq 4^{k}$. In addition, the conditions $X_{i+1} \neq \emptyset$ and $X_{1} \cap \ldots \cap X_{i} \neq \emptyset$ imply that $U$ contains a point $u^{\prime} \in X_{i+1}$ and $u^{\prime \prime} \in X_{1} \cap \ldots \cap X_{i}$. Then every cell in $C_{\Delta}^{0 \backslash} \backslash L_{i+1}$ is covered by the points $u^{\prime}, s$ or $u^{\prime \prime}, s$ for a suitable $s \in S$.

So we are concerned with the cells $C \in \mathscr{C}_{i+1}$ lying in $L_{i+1}$.

If $C \in \mathscr{C}_{i+1}^{q}$ or $C \in \mathscr{C}_{i+1}^{z}$, then the method of "either cover or augment" works in the same way as in Cases 1,2 and 3.

Finally, if $C \in \mathscr{C}_{i+1}^{x}$, then $C \subset C_{i} \subset C_{i-1} \subset \ldots \subset C_{1}$ where $C_{j} \in \mathscr{C}_{j}$. (This chain of cells exists and is unique because the subdivision of $L_{j+1}$ is a refinement of the 
subdivision of $L_{j}$, restricted to $L_{j+1}$.) But it cannot be the case that each $C_{j} \in \mathscr{C}_{j}^{x}$ because then $X_{i+1} \cap \ldots \cap X_{1} \supseteq V \cap\left(C \cap C_{i} \cap \ldots \cap C_{1}\right) \neq \emptyset$, a contradiction. So $C_{j} \in$ $\in \mathscr{C}_{j}^{y} \cup \mathscr{C}_{j}^{z}$ for some $j=1, \ldots, \vec{i}$.

If $C_{j} \in \mathscr{C}_{j}^{z}$, then $C_{j}$ is covered by a pair $u, s \in S$ where $u \in V$ is a point from the cell in $\mathscr{C}_{j}$ that covers $C_{j}$. The points $u, s$ cover then the cell $C$ as well.

If $C_{j} \in \mathscr{C}_{j}^{y}$, then the pair $C_{j},(S \cup\{u\})$ is a good pair for a suitable $u \in V \cap L_{j-1}$, and, a fortiori, $(C, S \cup\{u\})$ is a good pair as well.

So for each $C \in \mathscr{C}_{i+1}^{x}$ we pick one more point $u \in V \cap C_{\Delta}^{0}$ in such a way that either $C$ is covered by $u$ and some $s \in S$ or $(C, S \cup\{u\})$ is a good pair. The set of these points is $U_{0}$, clearly $\left|U_{0}\right| \leqq\left|\mathscr{C}_{i+1}^{x}\right| \leqq 1 / 4\left|\mathscr{C}_{i}\right|=4^{k-1}$.

What we did in Case $4 \mathrm{a}$ is a modification of the method "either cover or augment". This modification uses at most $|U|+\left|U_{0}\right| \leqq 5 \cdot 4^{k-1}$ points. So we see that in Cases $1,2,3$ and 4 a our method works using at most $5 \cdot 4^{k-1}$ points, while in Case $4 b$ we need only one point to cover every point in $C_{\Delta}^{0} \cap V$.

The number of steps being limited by $|S| \leqq 4 \pi \varepsilon^{-1}$ we conclude that Theorem 1 holds with

$$
n(\varepsilon)=\left(5 \cdot 4^{k-1}\right)^{4 \pi \varepsilon^{-1}} .
$$

The proof of Theorem 2 is almost identical with the previous one. The only differences are in Definition 2, Lemma 1 and 2. In Definition 2, (i) is to be modified to $\varrho(c, s) \geqq 10 \sqrt{d} \Delta \varepsilon^{-1}(\forall c \in C, \forall s \in S)$, in Lemma 1 we have only $|S| \leqq\left(c_{2} \varepsilon^{-1}\right)^{d-1}$, and in Lemma 2, condition $\varrho\left(c_{1}, c_{2}\right) \geqq 10 \sqrt{2} \Delta \varepsilon^{-1}$ has to be replaced by $\varrho\left(c_{1}, c_{2}\right) \geqq$ $\geqq 10 \sqrt{d} \Delta \varepsilon^{-1}$. In the proof, $k=k(\varepsilon)=\left[\log _{2}\left(200 \sqrt{d} \varepsilon^{-1}\right)\right]$ giving

$$
n(d, \varepsilon)=\left(\left(1+\frac{1}{2^{d}}\right) \frac{200 \sqrt{d}}{\varepsilon}\right)^{\left(2 \pi d^{3 / 2}\right)(4 / e)^{d-1}} .
$$

\section{Proof of Theorem 3}

As mentioned in the introduction, we will use the following

Lemma 3. If the origin belongs to conv $\left\{a_{0}, \ldots, a_{d}\right\}$ where $a_{0}, \ldots, a_{d} \in \mathbf{R}^{d}$, then there are indices $i$ and $j$ with $\Varangle\left(a_{i} 0 a_{j}\right) \geqq \pi-\operatorname{arc} \cos 1 / d$.

Proof. We may assume that $\left\|a_{0}\right\|=\left\|a_{1}\right\|=\ldots=\left\|a_{d}\right\|=1$ because we are concerned with angles at 0 . Assume indirectly, that $\left\langle a_{i}, a_{j}\right\rangle>-1 / d$ for each $0 \leqq i<j \leqq d$. The condition $0 \in \operatorname{conv}\left\{a_{0}, a_{1}, \ldots, a_{d}\right\}$ implies the existence of $\alpha_{0}, \ldots, \alpha_{d} \geqq 0$ with $\sum_{i=0}^{d} \alpha_{i}=1$ and

$$
0=\sum_{i=0}^{d} \alpha_{i} a_{i}
$$

Multiplying this by $a_{j}$ and using $\left\langle a_{i}, a_{j}\right\rangle>-1 / d$ we get

$$
-\left(1-\alpha_{j}\right) \frac{1}{d}=\sum_{\substack{i=0 \\ i \neq j}}^{d} \alpha_{i}\left(-\frac{1}{d}\right)<\sum_{\substack{i=0 \\ i \neq j}}^{d} \alpha_{i}\left\langle a_{i}, a_{j}\right\rangle=-\alpha_{j} .
$$


Summing these inequalities for $j=0, \ldots, d$ we have

a contradiction.

$$
-1=\sum_{j=0}^{d}-\left(1-\alpha_{j}\right) \frac{1}{d}<-\sum_{j=0}^{d} \alpha_{j}=-1
$$

The proof of Theorem 3 is by induction on $d$. The cases $d=1$ and $d=2$ are easy.

Assume the theorem holds in the $k$-dimensional space for $k<d$. We are going to prove it in the $d$-dimensional space.

As $V$ is finite, cony $V$ is a convex polytope. Denote the set of its vertices by $U$, the set of facets by $\mathscr{L}$, and the set of unit outer normals to the facets by $N$. So $N \subset S^{d-1}$ the unit sphere in $\mathbf{R}^{d}$. Take an $\varepsilon$-net $M \leqq S^{d-1}$ with

$$
|M| \leqq d\left(\frac{2}{\varepsilon}\right)^{d-1}
$$

$\varepsilon>0$ will be specified later. Set $\omega_{d}=\arccos (-1 / d)$.

Consider a point $m \in M$ and the set $N_{m}=\{n \in N:|n-m| \leqq \varepsilon\} . N_{m}$ is the set of outer normals to the facets $\mathscr{L}_{m}$ of conv $V$. Let $H_{m} \subseteq \mathbf{R}^{d}$ be a hyperplane with normal $m$. The projection $\pi_{m}$ (or $\pi$, for short) to $H_{m}$ maps the set $K_{m}=U\left(L: L \in \mathscr{L}_{m}\right)$ to $H_{m}$ that can be taken for $\mathbf{R}^{d-1}$. The vertex set $U_{m}$ of the polytope conv $K_{m}$ is clearly a subset of $U$, and $\pi\left(\operatorname{conv} K_{m}\right)=\pi\left(\operatorname{conv} U_{m}\right)=\operatorname{conv} \pi\left(U_{m}\right)$. So we may apply the induction hypothesis to the set $\pi\left(U_{m}\right) \subseteq H_{m}=\mathbf{R}^{d-1}$. Then we get a subset $W_{m} \subseteq \pi\left(U_{m}\right),\left|W_{m}\right| \leqq n(d-1)$ such that for any point $u \in\left(\right.$ conv $\left.\pi\left(U_{m}\right)\right) \backslash W_{m}$ there exist two points $w_{1}, w_{2} \in W_{m}$ with $\varangle\left(w_{1} u w_{2}\right) \geqq \omega_{d-1}$. It is clear that for each $u \in$ $\in \operatorname{conv} \pi\left(U_{m}\right)$ there is exactly one point $\hat{u} \in K_{m}$ with $\pi(\hat{u})=u$.

Now we show that $\varangle\left(\hat{w}_{1} \hat{u} \hat{w}_{2}\right) \geqq \omega_{d-1}-2 \varepsilon$ if $\hat{u} \in L$ for some $L \in \mathscr{L}_{m}$. For this end it is enough prove that if $w \in W_{m}$ then the angle between the lines $w u$ and $\hat{w} \hat{u}$ is at most $\varepsilon$ where $u=\pi(\hat{u})$ and $w=\pi(\hat{w})$. This implies the claim because then

$$
\left|\nless\left(\hat{w}_{1} \hat{u} \hat{w}_{2}\right)-\varangle\left(w_{1} u w_{2}\right)\right| \leqq 2 \varepsilon .
$$

If $w \in W_{m}$ then $\hat{w} \in L^{\prime}$ for some $L^{\prime} \in \mathscr{L}_{m}$. Pick a point $\hat{z} \in L^{\prime}$ from the relative interior of $L^{\prime}$ and very close to $\hat{w}$. Similarly, pick a point $\hat{v} \in L$ from the relative interior of $L$ and very close to $\hat{u}$. We prove that the angle between the lines through $\hat{z}$ and $\hat{v}$ and through $z$ and $v$ is at most $\varepsilon$ where $z=\pi(\hat{z})$ and $v=\pi(\hat{v})$. This is clearly sufficient.

Consider the two-dimensional plane $P$ spanned by $\hat{z}, \hat{v}, z$ and $v . P \cap$ conv $K_{m}$ is a convex polygon on the plane. The points $\hat{z}$ and $\hat{v}$ lie on its boundary. The angle between the tangent line to the polygon at $\hat{z}$ and the line $z v$ is at most $\varepsilon$, the same is true at $\hat{v}$. This follows from the definition of $N_{m}$. Observe that $\hat{z}$ and $\hat{v}$ are not on "opposite" sides of the polygon because the outer normals at them are near to each other. Then the slope of the chord $\hat{z} \hat{v}$ of the polygon is between the slopes of the tangent lines at $\varepsilon$ and $\hat{v}$. And this is what we wanted to prove.

Choose now $\varepsilon>0$ in such a way that $\omega_{d}=\omega_{d-1}-2 \varepsilon$. Set

$$
W=\bigcup_{m \in M} W_{m}=\left\{x \in U: \pi_{m}(x) \in W_{m}, x \in K_{m} \text { for some } m \in M\right\} \text {. }
$$


Then $|W| \leqq n(d-1) \cdot|M| \leqq n(d-1) \cdot 4^{d-1} d^{2 d-1}$, and for any point $u \in$ boundary (conv $V$ ) there exist two points $w_{1}, w_{2} \in W$ with $\Varangle\left(w_{1} u w_{2}\right) \geqq \omega_{d}$.

Moreover, $W$ "covers" also conv $W$ because by Carathéodory's theorem [3] conv $W$ is the union of $d$-dimensional simplices with vertices from $W$, and then we can apply Lemma 3.

So far we have proved that the theorem is true for any $u \in[$ conv $W \cup$ boundary (conv $V)] \backslash W$. So we pick a point $u \in \operatorname{conv} V$ with $u \notin$ conv $W \cup$ boundary (conv $V$ ). We are going to find two points $w_{1}, w_{2} \in W$ with $\varangle\left(w_{1} u w_{2}\right) \geqq \omega_{d}$.

Denote by $z$ the point in conv $W$, nearest to $u$. The halfine $z u$ (starting at $z$ ) meets boundary(conv $V$ ) in the point $v$. The hyperplane $H$ through $z$ with normal $u-z$ is a supporting hyperplane to $\operatorname{conv} W$, so the two points $w_{1}, w_{2} \in W$ with $\varangle\left(w_{1} v w_{2}\right) \geqq \omega_{d}$ lie on that side of $H$ which does not contain $v$, i.e.,

$$
\Varangle\left(w_{1} z v\right) \geqq \frac{\pi}{2}, \quad \varangle\left(w_{2} z v\right) \geqq \frac{\pi}{2} .
$$

Using this property it is easy to show that $\Varangle\left(w_{1} u w_{2}\right) \geqq \Varangle\left(w_{1} v w_{2}\right)$. We omit the details.

A simple computation shows now that

$$
n(d) \leqq 2^{d(d-1)} d^{d(d+1)} .
$$

\title{
References
}

[1] N. Alon, Z. Füredi and M. KATChalski, Separating pairs of points by standard boxes, European Journal of Combinatorics, 6 (1985), 205-210.

[2] I. BÁRANY and J. LEHEL, Covering with Euclidean boxes, submitted for publication.

[3] L. Danzer, B. Grünbaum and V. KleE, Helly's theorem and its relatives, in: Proc. of Symp. in Pure Mathernatics, VII. Convexity, AMS, Providence, 1963.

[4] P. ERDös and $Z$. Füredr, The greatest angle among $n$ points in the $d$-dimensional Euclidean space, Annals of Discrete Math. 17 (1983), 275-283.

[5] P. Erdös and G. SzeKeres, On some extremum problems in elementary geometry, Ann. Univ. Sci. Budapest, Eötvös Sect. Math. III/IV (1960-61), 53-62.

[6] P. ERDós and G. SzEKERES, A combinatorial problem in geometry, Compositio Math. 2 (1935), $463-470$.

[7] J. PACH, private communication.

\section{Bárány}

\author{
Mathematical Institute of the \\ Hungarian Academy of Sciences \\ H-1364, Budapest, P.O.B. 127 \\ Hungary
}

